\title{
Correction: Theory of diffusion-influenced reactions in complex geometries
}

Cite this: Phys. Chem. Chem. Phys., 2016, 18, 17757

\author{
Marta Galanti, ${ }^{\text {abcde }}$ Duccio Fanelli, ${ }^{\text {ac }}$ Sergey D. Traytak ${ }^{f}$ and Francesco Piazza*de \\ Correction for 'Theory of diffusion-influenced reactions in complex geometries' by Marta Galanti et al., \\ Phys. Chem. Chem. Phys., 2016, DOI: 10.1039/c6cp01147k.
} DOI: $10.1039 / \mathrm{c} 6 \mathrm{cp} 90149 \mathrm{~b}$

www.rsc.org/pccp

The affiliation details for Francesco Piazza are incorrect in the published article. The corrected details are given above. The Royal Society of Chemistry apologises for these errors and any consequent inconvenience to authors and readers.

\footnotetext{
${ }^{a}$ Università degli Studi di Firenze, Dipartimento di Fisica e Astronomia and CSDC, via G. Sansone 1, IT-50019 Sesto Fiorentino, Firenze, Italia

${ }^{b}$ Dipartimento di Sistemi e Informatica, Università di Firenze, Via S. Marta 3, 50139 Florence, Italy

${ }^{c}$ INFN, Sezione di Firenze, Italy

${ }^{d}$ Université d'Orléans, Château de la Source, 45100, Orléans, France. E-mail: Francesco.Piazza@gmail.com

${ }^{e}$ Centre de Biophysique Moléculaire, CNRS-UPR4301, Rue C. Sadron, 45071, Orléans, France

${ }^{f}$ Semenov Institute of Chemical Physics RAS, 4 Kosygina St., 117977, Moscow, Russia
} 